\title{
Implementation of a new junior-level optical engineering laboratory course at Rose-Hulman Institute of Technology
}

Paul Leisher, Sergio Granieri, Robert Bunch

Paul O. Leisher, Sergio C. Granieri, Robert M. Bunch, "Implementation of a new junior-level optical engineering laboratory course at Rose-Hulman Institute of Technology," Proc. SPIE 9946, Optics Education and Outreach IV, 99460S (27 September 2016); doi: 10.1117/12.2237164

EDent: SPIE Optical Engineering + Applications, 2016, San Diego, California, United States 


\title{
Implementation of a new junior-level optical engineering laboratory course at Rose-Hulman Institute of Technology
}

\author{
Paul O. Leisher, Sergio C. Granieri, and Robert M. Bunch \\ Rose-Hulman Institute of Technology, 5500 Wabash Ave., Terre Haute, IN, USA 47803
}

\begin{abstract}
The optical engineering program at Rose-Hulman recently developed a course titled "Optomechanics and Optical Engineering Lab." This course focuses on the design, assembly, and alignment of benchtop optical systems in an effort to expose students to the synthesis of concepts from the areas of geometrical optics, physical optics, photonic devices, and optomechanics. Minimal guidance is provided to the students regarding procedure - students must devise their own methodology and data collection/analysis plan. We present results from the implementation of this new course and details on the projects that the students carry out in the areas of spectroscopy, interferometry, photonics, and imaging.
\end{abstract}

Keywords: Optical engineering, curriculum development, laboratory experiments

\section{INTRODUCTION}

During the 2013-14 academic year we began a curriculum evaluation and revision of the B.S. (Optical Engineering) program at Rose-Hulman Institute of Technology [1-3]. This evaluation is a regular periodic process that we setup as part of our accreditation requirements of the Engineering Accreditation Commission (EAC) of ABET [3,4]. At that time, we also needed to address how our program planned to meet the specific program criteria for all Optical, Photonic, and Similarly Named Engineering Programs [4]. One of the issues that arose in evaluating our assessment data related to the laboratory preparation and the student's confidence in their laboratory skills when the students entered the capstone design course sequence. We also needed to explicitly provide instruction on the basics of opto-mechanics driven by the rearrangement of topics in other courses during the curriculum revision.

A common approach to teaching laboratory courses typically proceeds as follows: students are provided with a laboratory manual which details the steps needed to complete the lab; students carry out each experiment moving step-by-step through the provided procedure while keeping a live record of their procedure, data, and analysis in a laboratory notebook which is submitted for grading. While this is a useful approach during the freshman and sophomore year to allow students to become familiar with experimental processes and observe physical and optical phenomena, it does not expose students to the necessary skills needed in a capstone project. Our assessment showed that students did not have confidence in their ability to reason through each step in the procedure. Some students blindly followed the written steps and in many cases missed the entire point of the experiment.

We already use project activities and assignments in several courses and we have been especially successful using a projectbased approach in our engineering capstone design course sequence [2]. To better prepare the student for the capstone design experience course sequence we decided to develop a project-based laboratory course to be taken during a student's junior year. This paper describes the development of this course and some lessons learned in teaching this course. 


\section{COURSE OVERVIEW}

The new junior-level course, OE 395 - Optomechanics \& Optical Engineering Lab, is scheduled for first (fall) quarter of the junior year. The course is required for students seeking a B.S. degree in Optical Engineering. Enrollment in the course requires completion of three prerequisites: PH 292 - Physical Optics, OE 280 - Geometrical Optics, and OE 295 Photonics Devices and Systems. The primary objective of the course is to teach students, in a hands-on laboratory environment, how topics from these seemingly disconnected areas of optics actually come together in real optical designs. The course meets a total of four times per week over the 10 week academic quarter: two 1-hour lectures and two 3-hour lab periods. The full course description as it appears in the Rose-Hulman course catalog is shown below.

OE 395 - Optomechanics \& Optical Engineering Lab - Design, assembly, and alignment of bench top optical systems. Introduction to experimental techniques in optics. Data collection and analysis. Relevant lecture topics including principles of opto-mechanical design, fold mirrors and prisms, lens and mirror mounting, kinematic mounts, precision adjustments and control.

An introduction to the principles of optomechanics are covered during the lecture periods. Homework problems are assigned to reinforce concepts introduced in the lecture and two exams (not comprehensive) are scheduled during the academic quarter to assess student learning. Student performance in this portion of the class represents $40 \%$ of their final grade. Topics covered in the lecture portion of the class include:

- Alignment, effects of motions, beam shifts, line-of-sight, image position errors, curvature errors, tilt elements with power-error compilations

- Folding mirrors, image orientations, parity, matrix formalism for ray tracing in mirror systems, wedges, prisms and prism unfolding

- Optical component specifications, ISO drawings, layouts

- Kinematic mounts and precision alignment, lens mounting, sizing, tolerances, centering, preload, environmental/thermal effects, and athermalization.

The main emphasis of the course, however, is on the laboratory experience. A series of introductory directed "refresher" experiments are completed in the first week to help the students who are just returning from their summer break get back into the lab. In the first of these, the students are required to align two mirrors to direct a collimated HeNe beam through a drinking straw placed at a random angle $(\theta$ and $\varphi)$ with respect to the normal of the of the optical table. In the second experiment, the students are required to set up a spatial filter with the HeNe laser to produce a clean high-quality diffraction limited beam (which could be used for interferometry). The third experiment involves setting up and programming a basic Arduino development board to control the output of an LED and input the received signal from a photodetector.

Following completion of the introductory experiments, the students begin work on the design projects which comprise the bulk of their laboratory efforts. Four projects in total were developed and are intended to expose the students to topics spanning multiple areas of optical engineering. Table 1 depicts the mapping of the four projects to the four areas of optical engineering specifically designated in the ABET program-specific criteria for Optical Engineering, Photonic Engineering, and similarly named programs [4], and each is discussed further in Section 3.

\begin{tabular}{|l|c|c|c|c|}
\hline & $\begin{array}{c}\text { Geometrical } \\
\text { Optics }\end{array}$ & $\begin{array}{c}\text { Physical } \\
\text { Optics }\end{array}$ & $\begin{array}{c}\text { Photonic } \\
\text { Devices }\end{array}$ & $\begin{array}{c}\text { Optical } \\
\text { Materials }\end{array}$ \\
\hline Project \#1 - Absorption Spectroscopy & $\checkmark$ & & $\checkmark$ & $\checkmark$ \\
\hline Project \#2- Refractive Index of Air & $\checkmark$ & $\checkmark$ & $\checkmark$ & $\checkmark$ \\
\hline Project \#3 - Speed of Light & $\checkmark$ & & $\checkmark$ & \\
\hline Project \#4 - Camera Lens & $\checkmark$ & $\checkmark$ & & \\
\hline
\end{tabular}

Table 1 - Mapping of the four OE 395 projects to the four main areas of optical engineering. Successful completion of each project relies on the synthesis of concepts from several of the areas. 
Students taking the course are required to keep a laboratory notebook, though the format is not prescribed. The laboratory notebook is reviewed by the course instructors upon the completion of each project and feedback is provided. These notebook checks account for $25 \%$ of the students' final course letter grade. At the end of the academic quarter, a final report is assigned which each student is required to complete on an individual basis. The final report is a manuscript-style (formatted to SPIE specifications) full accounting of a single laboratory project from the academic quarter. The students are not told which project they will need to report on until the last week of class. This approach provides a realistic scenario of what would happen in the field and encourages the students to maintain a well-documented notebook, with complete diagrams, data sets, plots, photographs, and analysis.

Each group of two students is provided a kit containing of various optical and opto-mechanical components from which their setup must be designed. The kits include optical posts, post holders, lens and mirror mounts, lens tubes, kinematic translation and rotation stages, uncoated spherical lenses of various focal lengths, aspheric objective lenses, flat and spherical mirrors, diffraction gratings, filters, beamsplitters, apertures, irises, a switchable gain Si photodetector, and a CCD camera. The components for these kits were selected by the faculty to provide a wide diversity of possible solutions to the experimental projects the students are expected to complete. A photograph of one of the kits is shown in Figure 1 and a complete listing of the kit contents is provided in Table 2. All parts were supplied by Thorlabs, Inc.[5] and the total cost for each kit is $\sim \$ 5000$. Optical sources included $5 \mathrm{~mW}$ HeNe lasers, $5 \mathrm{~mW} 635 \mathrm{~nm}$ diode laser sources, and halogen which light sources are also provided for use, and instrumentation such as oscilloscopes, digital multimeters, and computers are also made available.

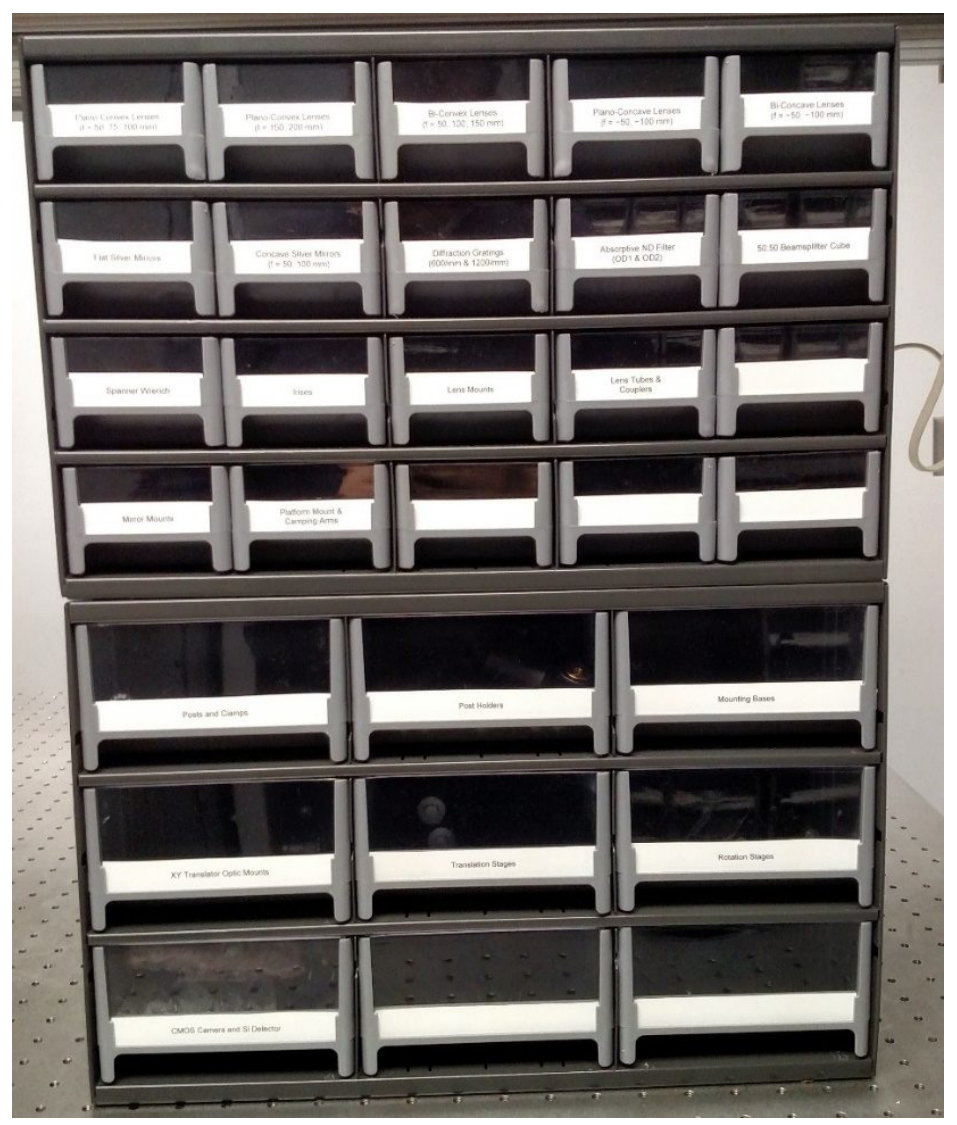

Figure 1 - Photograph of one optical component kit provided to a student team in OE 395. 


\begin{tabular}{|c|c|c|}
\hline Thorlabs Part \# & Description & Qty \\
\hline TR3-P5 & Ø1/2" Optical Post, 8-32 Setscrew, 1/4"-20 Tap, L = 3", 5 Pack & 2 \\
\hline TR1.5-P5 & $\varnothing 1 / 2 "$ Optical Post, 8-32 Setscrew, 1/4"-20 Tap, L = 1.5", 5 Pack & 2 \\
\hline RA90 & Right-Angle Post Clamp, Fixed $90^{\circ}$ Adapter & 2 \\
\hline RA180 & Post End Clamp, 1/4"-20 Stud and Fixed $90^{\circ}$ Clamp & 2 \\
\hline SWC & Rotating Clamp for $\varnothing 1 / 2 "$ Posts, $360^{\circ}$ Continuously Adjus table, 3/16" Hex & 2 \\
\hline PH3-P5 & Ø1/2" Post Holders, Spring-Loaded Hex-Locking Thumbscrew, L = 3", 5 Pack & 2 \\
\hline PH1.5-P5 & $\varnothing 1 / 2 "$ Post Holders, Spring-Loaded Hex-Locking Thumbscrews, L=1.5", 5 Pack & 2 \\
\hline $\mathrm{BA} 2$ & Mounting Base, 2" x3" ×3/8" (50 mm x 75 mm x $10 \mathrm{~mm})$ & 5 \\
\hline BA1 & Mounting Base, 1" ×3" ×3/8" (25 mm x 75 mm x $10 \mathrm{~mm})$ & 5 \\
\hline ST1XY-S & XY Translator with Micrometer Drives & 2 \\
\hline PT1 & 1" Translation Stage with Standard Micrometer, 1/4"-20 Taps & 3 \\
\hline PR01 & High-Precision Rotation Mount, One SM1RR Retaining Ring Included & 1 \\
\hline PR01A & Solid Adapter Plate for PR01, Four 4-40 Cap Screws Included & 1 \\
\hline PDA36A & Si Switchable Gain Detector, $350-1100 \mathrm{~nm}, 10 \mathrm{MHz}$ BW, $13 \mathrm{~mm} 2,120 \mathrm{VAC}$ & 1 \\
\hline DCC1545M & DCC1545M High Resolution USB2.0 CMOS Camera, $1280 \times 1024$, Monochrome Sensor & 1 \\
\hline LA1131 & N-BK7 Plano-Convex Lens, $\varnothing 1 ", \mathrm{f}=50.0 \mathrm{~mm}$, Uncoated & 2 \\
\hline LA1608 & N-BK7 Plano-Convex Lens, Ø1", $\mathrm{f}=75.0 \mathrm{~mm}$, Uncoated & 2 \\
\hline LA1509 & N-BK7 Plano-Convex Lens, Ø1", $\mathrm{f}=100.0 \mathrm{~mm}$, Uncoated & 2 \\
\hline LA1433 & N-BK7 Plano-Convex Lens, Ø1", $\mathrm{f}=150.0 \mathrm{~mm}$, Uncoated & 2 \\
\hline LA1708 & N-BK7 Plano-Convex Lens, Ø1", $\mathrm{f}=200.0 \mathrm{~mm}$, Uncoated & 2 \\
\hline LB1471 & N-BK7 Bi-Convex Lens, $\varnothing 1 ", f=50.0 \mathrm{~mm}$, Uncoated & 1 \\
\hline LB1676 & N-BK7 Bi-Convex Lens, $\varnothing 1 ", \mathrm{f}=100.0 \mathrm{~mm}$, Uncoated & 1 \\
\hline LB1437 & N-BK7 Bi-Convex Lens, $\varnothing 1 ", \mathrm{f}=150.0 \mathrm{~mm}$, Uncoated & 1 \\
\hline LC1715 & N-BK7 Plano-Concave Lens, $\varnothing 1 ", f=-50.0 \mathrm{~mm}$, Uncoated & 1 \\
\hline LC1120 & N-BK7 Plano-Concave Lens, $\varnothing 1 ", \mathrm{f}=-100.0 \mathrm{~mm}$, Uncoated & 1 \\
\hline LD1464 & N-BK7 Bi-Concave Lens, $\varnothing 25.4 \mathrm{~mm}, \mathrm{f}=-50.0 \mathrm{~mm}$, Uncoated & 1 \\
\hline LD1613 & N-BK7 Bi-Concave Lens, $\varnothing 25.4 \mathrm{~mm}, \mathrm{f}=-100.0 \mathrm{~mm}$, Uncoated & 1 \\
\hline PF10-03-P01 & $\varnothing 1 "$ (Ø25.4 mm) Protected Silver Mirror, 0.24" (6.0 mm) Thick & 4 \\
\hline CM254-050-P01 & $\varnothing 1 "$ Silver-Coated Concave Mirror, $\mathrm{f}=50.0 \mathrm{~mm}$ & 2 \\
\hline CM254-100-P01 & $\varnothing 1 "$ Silver-Coated Concave Mirror, $\mathrm{f}=100.0 \mathrm{~mm}$ & 2 \\
\hline GR25-0605 & Ruled Diff. Grating, $600 / \mathrm{mm}, 500 \mathrm{~nm}$ Blaze, $25 \times 25 \times 6 \mathrm{~mm}$ & 1 \\
\hline GR25-1205 & Ruled Diff. Grating, $1200 / \mathrm{mm}, 500 \mathrm{~nm}$ Blaze, $25 \times 25 \times 6 \mathrm{~mm}$ & 1 \\
\hline NE10A & $\varnothing 25 \mathrm{~mm}$ Absorptive ND Filter, SM1-Threaded Mount, Optical Density: 1.0 & 1 \\
\hline NE20A & $\varnothing 25 \mathrm{~mm}$ Absorptive ND Filter, SM1-Threaded Mount, Optical Density: 2.0 & 1 \\
\hline BS013 & 50:50 Non-Polarizing Beams plitter Cube, 400 - 700 nm, 1" & 1 \\
\hline SPW602 & SM1 Spanner Wrench, Graduated, Length $=3.88$ & 1 \\
\hline SM1D12 & SM1 Lever-Actuated Iris Diaphragm $(\varnothing 0.8-\varnothing 12 \mathrm{~mm})$ & 3 \\
\hline LMR1 & Imperial Lens Mount For 1" Optics, 8-32 Tap & 5 \\
\hline SM1L05 & SM1 Lens Tube, 0.5" Thread Depth, One Retaining Ring Included & 2 \\
\hline SM1L10 & SM1 Lens Tube, 1" Thread Depth, One Retaining Ring Included & 2 \\
\hline SM1L20 & SM1 Lens Tube, 2" Thread Depth, One Retaining Ring Included & 1 \\
\hline SM1L20 & SM1 Lens Tube, 2.00" Thread Depth, One Retaining Ring Included & 1 \\
\hline SM1L40 & SM1 Lens Tube, 4.00" Thread Depth, One Retaining Ring Included & 1 \\
\hline SM1M10 & SM1 Lens Tube Without External Threads, 1" Long, Two Retaining Rings Included & 1 \\
\hline SM1T1 & SM1 (1.035"-40) Coupler, Internal Threads & 1 \\
\hline SM1T10 & SM1 (1.035"-40) Coupler, External Threads, 1" Long & 1 \\
\hline E09RMS & SM1 to M9 x 0.5 Lens Cell Adapter & 1 \\
\hline S1TM09 & Extended RMS to M9 $\times 0.5$ Adapter & 1 \\
\hline C230TMD-A & $\mathrm{f}=4.51 \mathrm{~mm}, \mathrm{NA}=0.55$ Mounted Geltech Aspheric Lens, $\mathrm{AR}: 350-700 \mathrm{~nm}$ & 1 \\
\hline C220TMD-A & $\mathrm{f}=11.00 \mathrm{~mm}, \mathrm{NA}=0.25$, Mounted Geltech Aspheric Lens, AR: $350-700 \mathrm{~nm}$ & 1 \\
\hline SP301 & Spanner Wrench for an M9 $\times 0.5$ Optics Housing, Length = 1" & 1 \\
\hline SM1A3 & Adapter with External SM1 Threads and Internal RMS Threads & 1 \\
\hline$M-40 x$ & Microscope Objective Lens, 40x, $0.65 \mathrm{NA}, 4.5 \mathrm{~mm}$ Focal Length & 1 \\
\hline POLARIS-K1 & Polaris ${ }^{\mathrm{TM}}$ Low Drift $\varnothing 1 "$ Kinematic Mirror Mount, 3 Adjusters & 2 \\
\hline KM100S & Kinematic Mount for 1" (25.4 mm) Tall Rectangular Optics, Right Handed & 1 \\
\hline KM100B & 2" x 2" Kinematic Platform Mount & 1 \\
\hline PM4 & Large Adjustable Clamping Arm, 6-32 Threaded Post & 2 \\
\hline S19909 & 9 Drawer Stackable Cabinet & 1 \\
\hline S19320 & 20 Drawer Stackable Cabinet & 1 \\
\hline TC2 & 20-Piece Balldriver and Hex Key Kit with Stand, Imperial & 1 \\
\hline
\end{tabular}

Table 2 - Complete listing of the contents of the kit provided to the students in the course.

Proc. of SPIE Vol. 994699460 S-4 


\section{LABORATORY PROJECTS}

\subsection{Project \#1 - Absorption Spectroscopy}

The absorption spectroscopy project was developed to combine concepts in first-order geometrical optics design with diffraction effects studied in physical optics. The project assignment, as provided to the students, is detailed in Table 3. The students are presented with a problem of measuring the absorption spectrum of a common organic dye. Note in the project assignment, very little guidance is provided on how the students might best to utilize the kit components to build a functioning system. Specifications such as wavelength accuracy and necessary bandwidth are left vague, and students are expected to develop appropriate specifications in response. Each team is expected to develop a first-order model to estimate their system bandwidth by applying the principles of geometrical optics combined with the grating equation. Students expend significant effort researching basic spectrometer designs and understanding the role first-order design choices (such as entrance and exit aperture sizes and lens/mirror focal lengths) have on the resolution of the system.

Absorption Spectroscopy Project Assignment
Rhodamine 6G (R6G) is a common organic dye used in a liquid solution as the gain medium of a
dye laser. The electronic states of the R6G molecule are split into several vibrational levels, each
further split into several rotational levels. These individual transition lines are broadened when the
molecule is in solution due to collisions and electrostatic perturbations. This gives rise to a near
continuous absorption spectrum in the visible wavelength range.
For this experiment, design and build a grating spectrometer and use it to characterize the relative
absorption of R6G dye in solution. The absorption spectrum can be obtained by comparing the
measured spectrum of a reference white light source before and after filling a cuvette with the R6G
dye. You may use a HeNe laser to calibrate the wavelength and estimate the resolution bandwidth
of your spectrometer. Be sure to compare these results with your first-order design estimates.
Finally, compare your measured spectrum with that obtained using a commercial spectrophotometer.

Table 3 - Absorption spectroscopy project assignment.

Figures 2 and 3 present sample work taken from one team's project notebook. Figure 2 depicts a schematic layout of the team's final optical design. The team used use a hand-made paper slit as the entrance slit for the system. A $100 \mathrm{~mm}$ effective focal length lens collimates the light from the plane of the slit and directs it onto the diffraction grating. Two 50 $\mathrm{mm}$ lenses provided in the kit were combined to serve as a $25 \mathrm{~mm}$ EFL focusing lens (which was not available in the kit) to image the dispersed slit on the CCD camera serving as a quasi-1D detector array for the spectral measurement.

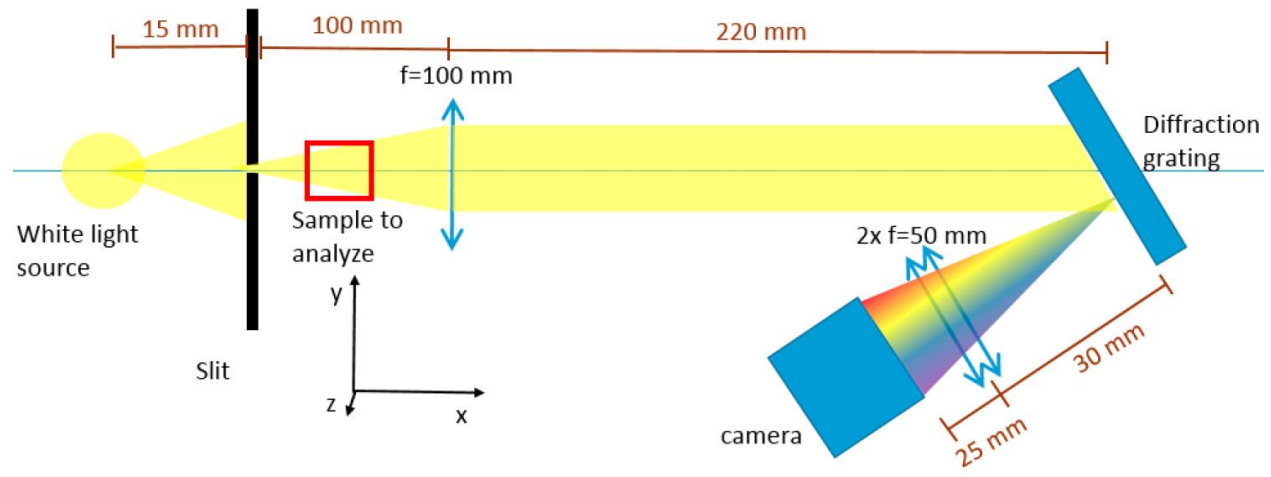

Figure 2 - (Project \#1 sample student work) Schematic layout of the camera-based imaging spectrometer used to measure the absorption spectrum of R6G dye. 
Figure 3 (Left) depicts a photograph of the same team's final setup. It is worth noting that this team was the only to use the CCD camera; the other three project teams this year elected to instead utilize the single photodetector with exit slit, and by rotating the grating, scan individual wavelengths. We believe that the approach of providing minimal guidance in the project assignment gives students the experiences exploring a diverse solution space. At right in Figure 4 is the team's measured transmission spectrum. These results were achieved by analyzing an image file output by the camera to determine the line intensity profile of one row of pixels.
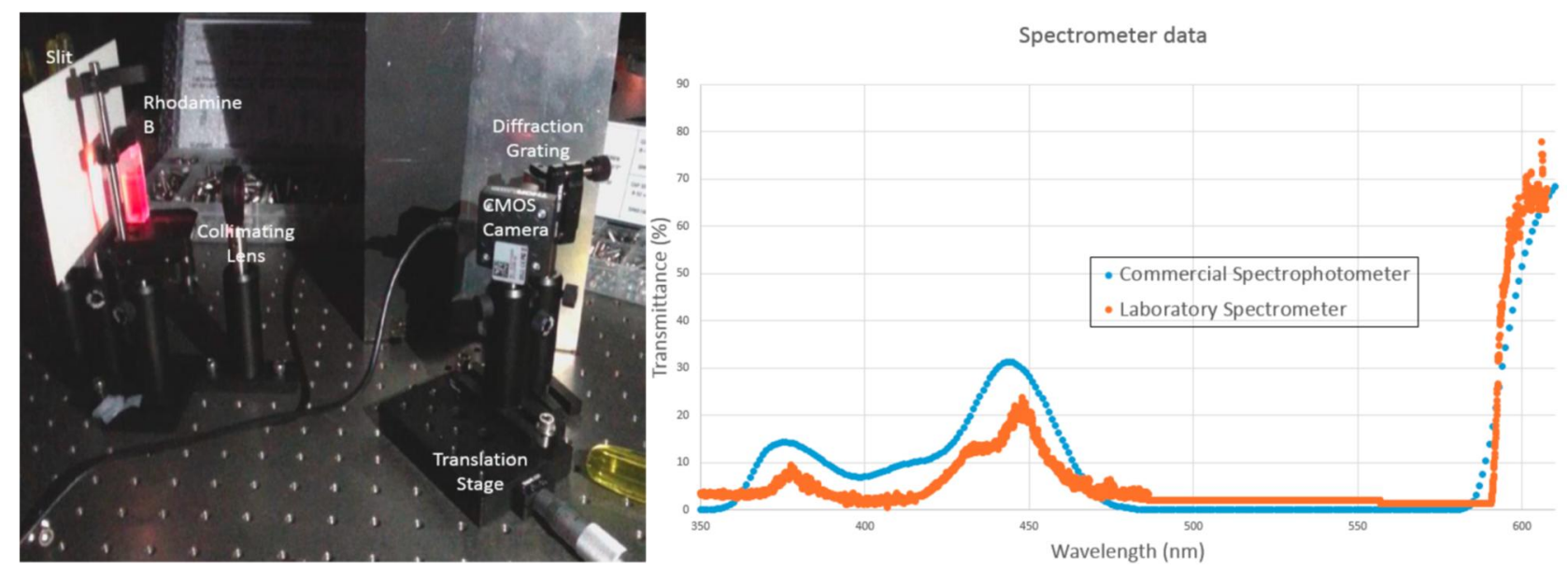

Figure 3 - (Project \#1 sample student work) (Left) Photograph of the benchtop absorption spectroscopy setup. (Right) Transmission spectrum measured in the students' spectrometer compared to results obtained in a commercial spectrophotometer.

Several issues were encountered during the execution of this first project. None of the groups directly measured the resolution of their system to compare to the calculated values. While this wasn't explicitly stated in the project statement, such verification of calculations is commonly expected of design engineers and in this case, it provided an excellent learning opportunity. Only one of the four teams actually developed a proper calibration process (wherein a source of known wavelength was measured to confirm wavelength calibration). Several groups failed to provide a plot directly comparing their output to the output of the spectrophotometer. These shortcomings led to a debriefing process which was implemented after each of the subsequent projects. In each case, the student teams discussed areas of potential improvement, missing results, and better methods for presenting their design results in a clear manner.

\subsection{Project \#2 - Refractive Index of Air}

The goal of the second project was to develop an experimental instrument to measure the refractive index of air. This project draws on the fundamentals of interferometry (a central topic to physical optics) combined with first-order optical design in order to achieve an appropriate result. Each student team developed a Michelson interferometer of varying dimensions and inserted an optical cell in one branch which could be evacuated. A photodetector at the exit plane is was commonly used by the student teams to assist in the counting of interference fringe changes as air is allowed to slowly bleed back into the optical cell. With knowledge of the cell dimensions and number of fringe changes between the evacuated cell and air-filled cell, the refractive index of air can be calculated with remarkable precision. While the general technique is clearly described, the development of appropriate mathematical model and complete optical design is again left completely up to the student. Table 4 provides the complete project assignment. 


Refractive Index of Air Project Assignment
The object of this experiment is to measure the refractive index of air using interferometery. Your task is to
construct an appropriate interferometer that will contain a gas cell in an arm of the interferometer. The difference
in the number of fringes displaced when the cell is evacuated to that when the cell is filled with air allows you
to calculate the index of refraction of air. Develop an appropriate theory for obtaining the value of the refractive
index based on your interferometer type and the measurement data that you will obtain. Be sure to include a
complete error analysis when reporting your final results.

Table 4 - Refractive index of air project assignment.

Figures 4 and 5 present sample work taken from one team's project notebook. A schematic diagram of the basic optical layout and as well as a photograph of the final setup for the team is shown in Figure 4. This team utilized a HeNe laser without the use of a spatal filter as the source. A beam expansion lens is used to adjust the collimated beam diameter at the detector plane. The beam is split (50:50) and each leg is reflected off a planar mirror. The careful alignment necessary to achieve a clear centered interfernce pattern at the detector plane was one of the more difficult aspects of the project. All four teams worked on the same optical bench making unintentional vibrations caused by others difficult to overcome.
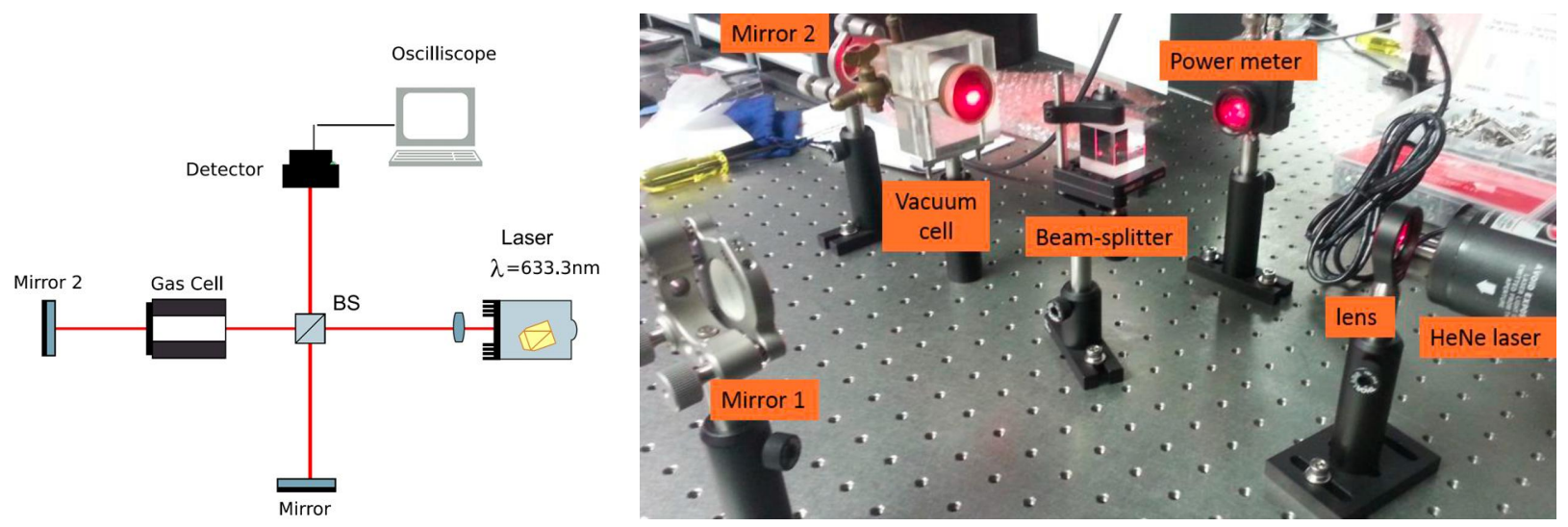

Figure 4 - (Project \#2 sample student work) (Left) Schematic layout and (right) photograph of the interferometer setup used in the refractive index of air experiment.

This project team monitored the photodetector output on an oscilloscope set to capture data over a long time period. The cell was first evacuated then data capture was started and a valve was slightly opened to allow air to slowly refill the cell (this approach eliminated the vibrations caused by the vacuum pump which caused significant noise in the measurement). Figure 5 presents one of the photodetector measurements vs. time (note the students terminated this test early, and likely failed to count the proper number of fringes). The team's data table from several tests is shown at right in Figure 5, with $\mathrm{N}$ denoting the number of fringes counted, and $\mathrm{n}$ referring to the refractive index of air at room temperature. The final reported value for the refractive index of air by this team was 1.00018 while the accepted value at room temperature is 1.00028; the student team correctly identified that the pump used in the experiment was unable to maintain a sufficiently low pressure for closer agreement. 


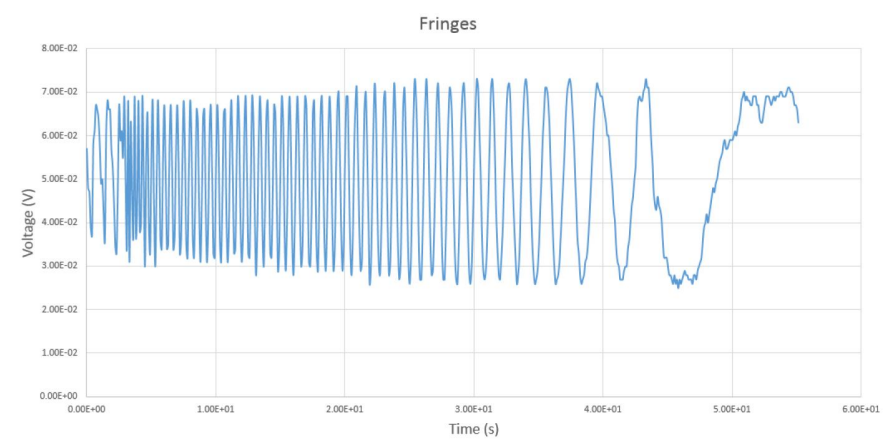

\begin{tabular}{|c|c|c|c|c|}
\hline Trial & $\mathrm{N}$ & $\mathrm{n}$ & & uncertainty \\
\hline 1 & 53 & 1.0001823 & \pm & 0.0000012 \\
\hline 2 & 54 & 1.0001858 & \pm & 0.0000011 \\
\hline 3 & 53 & 1.0001823 & \pm & 0.0000012 \\
\hline 4 & 53 & 1.0001823 & \pm & 0.0000012 \\
\hline 5 & 53 & 1.0001823 & \pm & 0.0000012 \\
\hline 6 & 52 & 1.0001789 & \pm & 0.0000011 \\
\hline 7 & 47 (incomplete) & & NA \\
\hline \multicolumn{6}{|c|}{ Average } & 1.00018232 & & $1.1667 \mathrm{E}-06$ \\
\hline
\end{tabular}

Figure 5 - (Project \#2 sample student work) (Left) Oscilloscope output from one trial run. (Right) Data table and calculation results for the refractive index of air.

\subsection{Project \#3 - Speed of Light}

In the third project, the students measured the speed of light in air using a direct time-of-flight based technique. This approach relies on the linear relationship between the observed phase shift modulated diode laser beam at the receiver and the distance through which the collimated beam travels before striking a photodetector. This experiment combines topics from the photonics (laser diode sources, detectors) with practical aspects of geometrical optics (such as achieving a sufficient degree of collimation of the beam to detect light after a significant propagation distance). The experiment is further constrained in that the students are limited to a work area approximately $5 \mathrm{ft} \times 2 \mathrm{ft}$ in dimension. Table 4 lists the project assignment as provided to the students.

Speed of Light Project Assignment
The object of this experiment is to measure the speed of light in air by direct time-of-flight
measurement. A modulated laser diode can be collimated and propagated through some distance
before being detected by a photodetector. By measuring the phase shift of the received signal as
function of the propogation distance, the speed of light in air can be deduced. One critical aspect to
this measurement will be ensuring the components (laser, detector) and instrumet (oscilloscope) are
sufficiently fast to perform this kind of measurement. Be sure to include error anlaysis in your
reporting.

Table 5 - Speed of light project assignment.

Figures 6 and 7 present sample work taken from one team's project notebook. At left in Figure 4 is a schematic drawing of the students' experimental setup and at right is a photograph of the oscillsocope showing the modulated source voltage and the photodetector output. One o
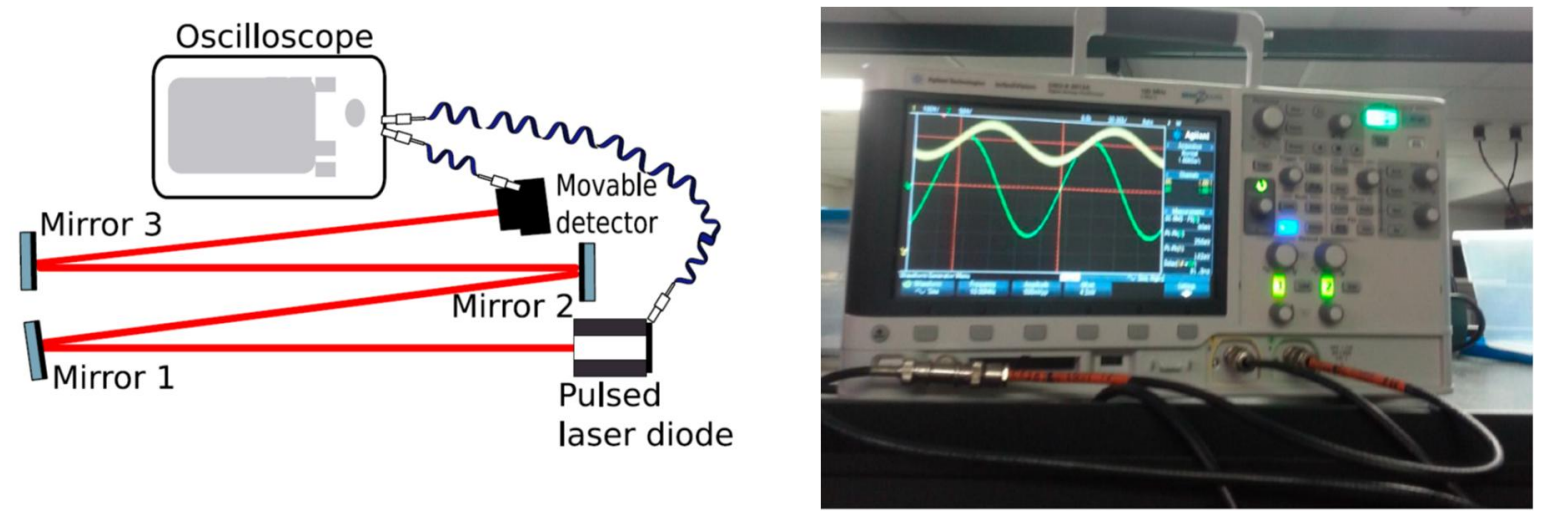

Figure 6 - (Project \#3 sample student work) (Left) Schematic layout and (Right) photograph of the experimental setup. 
Figure 7 depicts a plot of the propagation distance as a function of the measured phase delay. The slope of the line (in this case $2.97 \pm 0.08 \times 10^{8} \mathrm{~m} / \mathrm{s}$ ) gives the speed of light. One of the primary issues encountered by several teams in this experiment was a failure to identify that the phase shift at zero distance would still be nonzero. Several teams incorrectly calculated the speed of light from a single point measurement - dividing the propagation distance by the observed phase difference at that point.

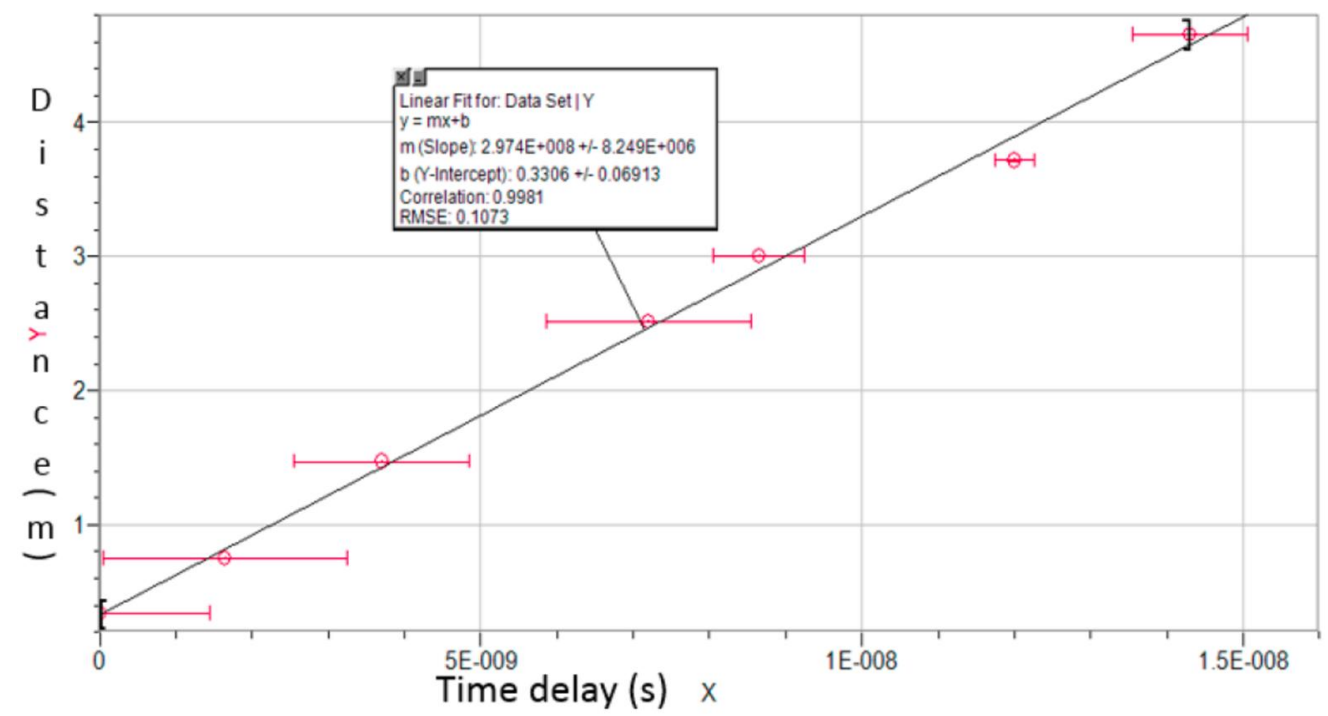

Figure 7 - (Project \#3 sample student work) Experimental data showing propagation distance vs. measured phase delay. The slope of the line gives the speed of light.

\subsection{Project \#4 - Camera Lens}

The last project in this course is the design and construction of a lens system for the ThorLabs DCC1545M CMOS camera. As shown in Table 6, there are several specifications that must be met and tested related to camera field-of-view but the design is open-ended with numerous possible solutions. The major constraint is that the lenses used in the design must be parts from the lab kit. Figure 8 shows a pictorial representation of the layout and a photograph of one of the student's designs. It is important to note that all student groups did perform a first-order layout in their preliminary design calculations. We decided to ask for contrast transfer function (CTF) measurements rather than a modulation transfer function (MTF) to simplify the experiment and to allow for students to identify and specify a test target (Figure 9). The camera software application allows for line profiles to be captured from an image and this is what was used to obtain data for the CTF as shown in Figure 10 with the resulting CTF of Figure 11. All students in the class appreciated the synthesis of topics from physical optics, geometrical optics, and photonic devices courses.

\section{Camera Lens Assignment}

In this experiment, you will build a lens system for the CMOS camera provided in your lens kit. The lens should be focused for objects far away from the camera. The half-field-of-view along the diagonal of the sensor should be $7.5^{\circ}$ (that is, $\theta^{1 / 2}= \pm 7.5^{\circ}$ ). You should devise a test to verify that your lens meets the field-of-view requirement. Assess the image quality of your design near the center of the field of view for aperture settings of $F / 3, F / 6$, and $F / 12$. The image quality should be quantified by means of direct measurement of the contrast transfer function vs. spatial frequency (one plot for each aperture setting). This will require you to design and print your own test target(s).

Table 6 - Camera lens assignment. 

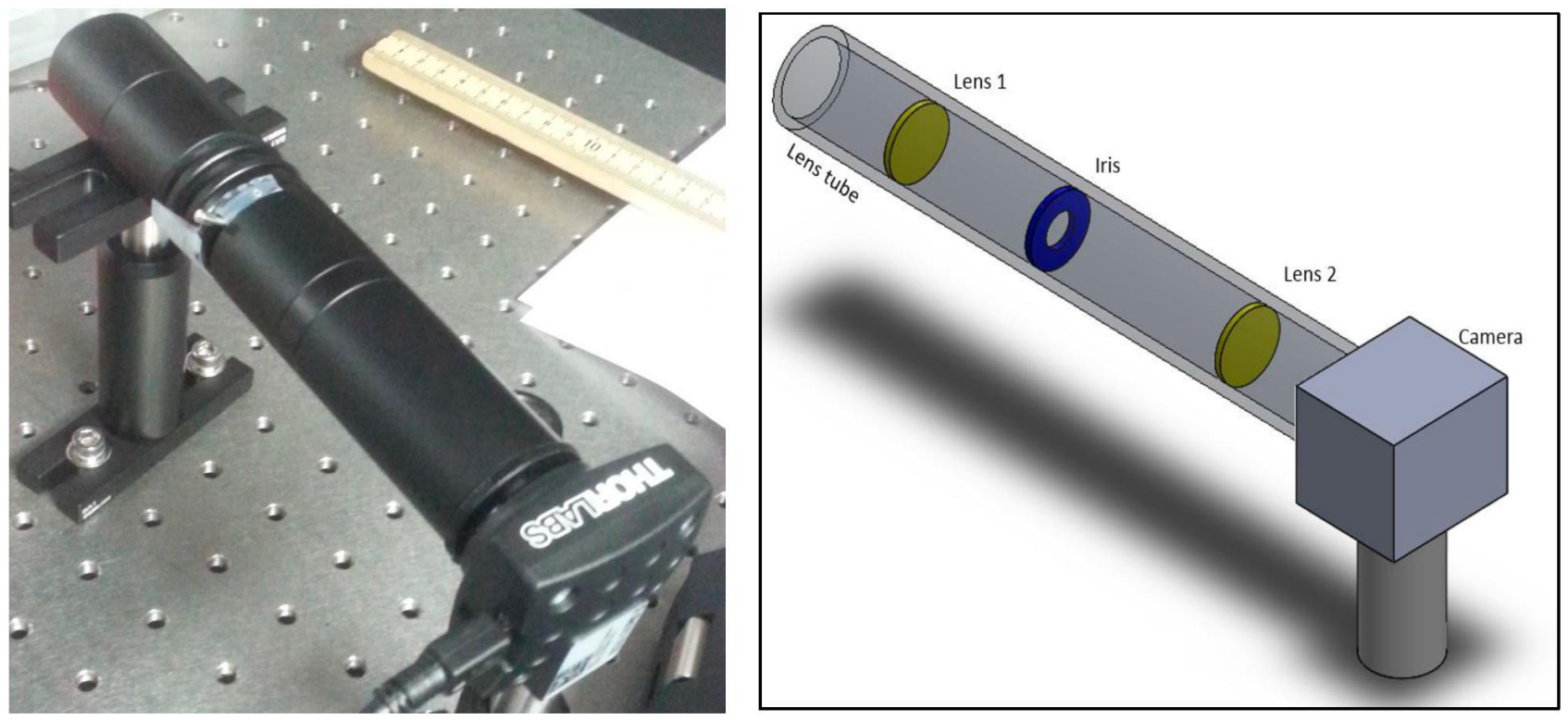

Figure 8 - (Project \#4 sample student work) (Left) Photograph and (Right) schematic layout of the compound lens.

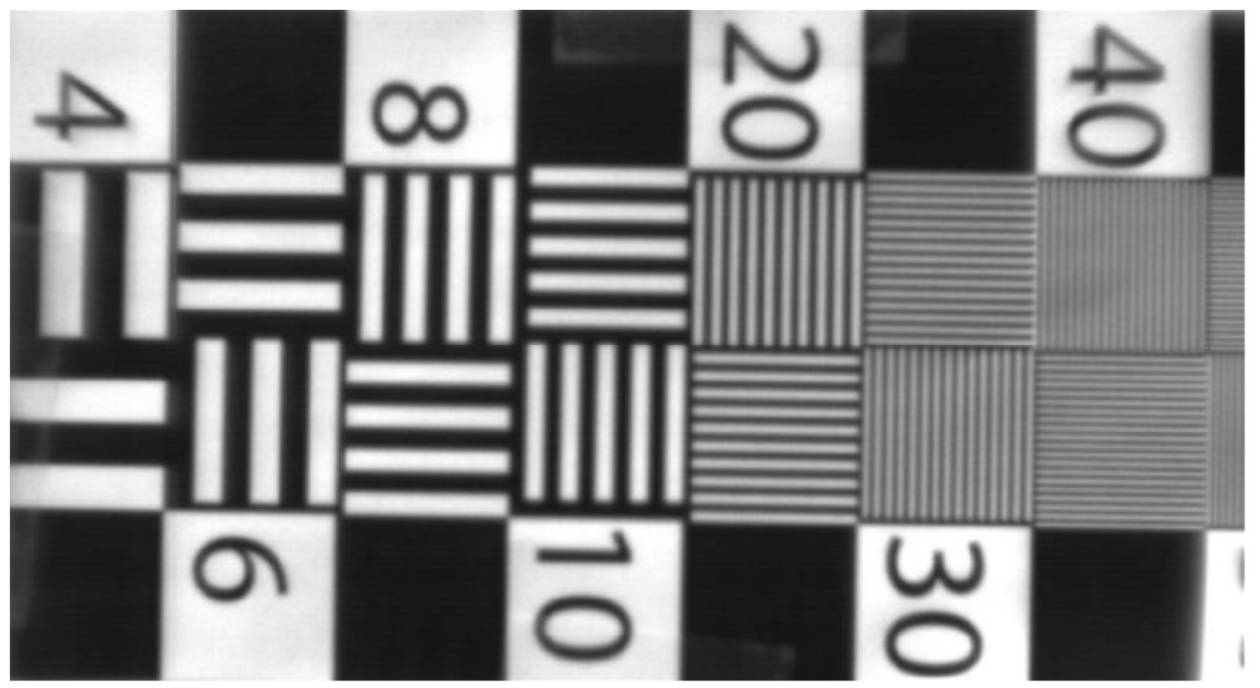

Figure 9 - (Project \#4 sample student work) Photograph of the contrast transfer function test chart taken with the compound camera lens. 


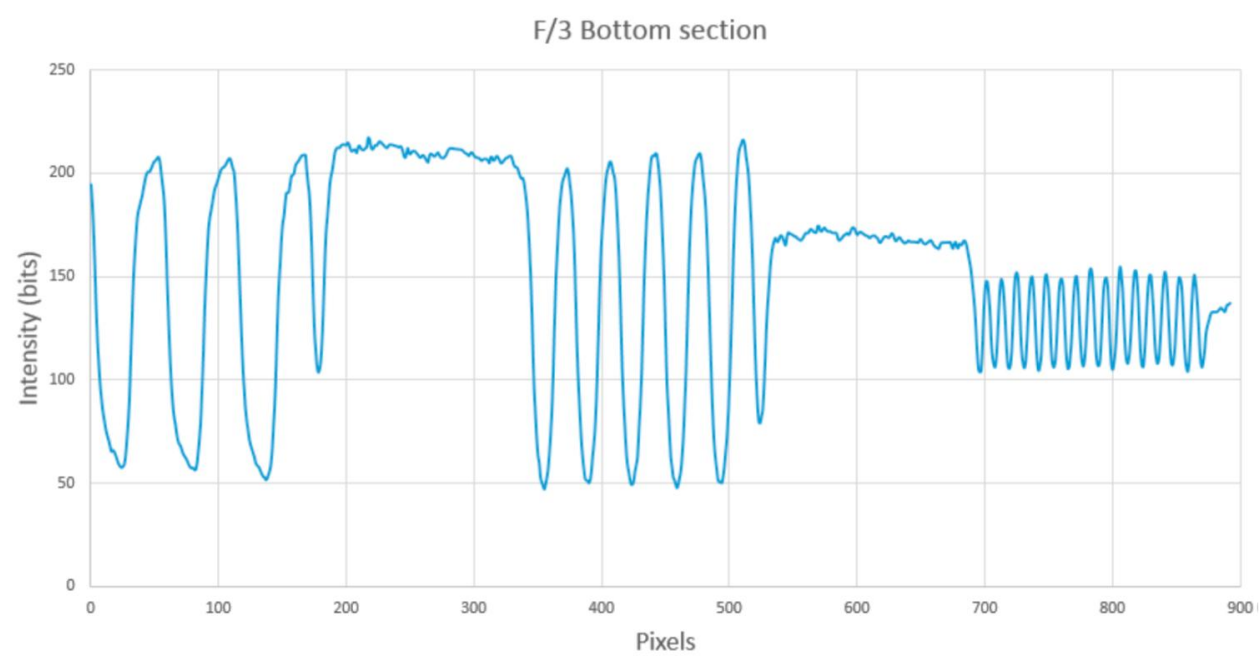

Figure 10 - (Project \#4 sample student work) Line profile generated from the contrast transfer function test chart image shown in Figure 8.

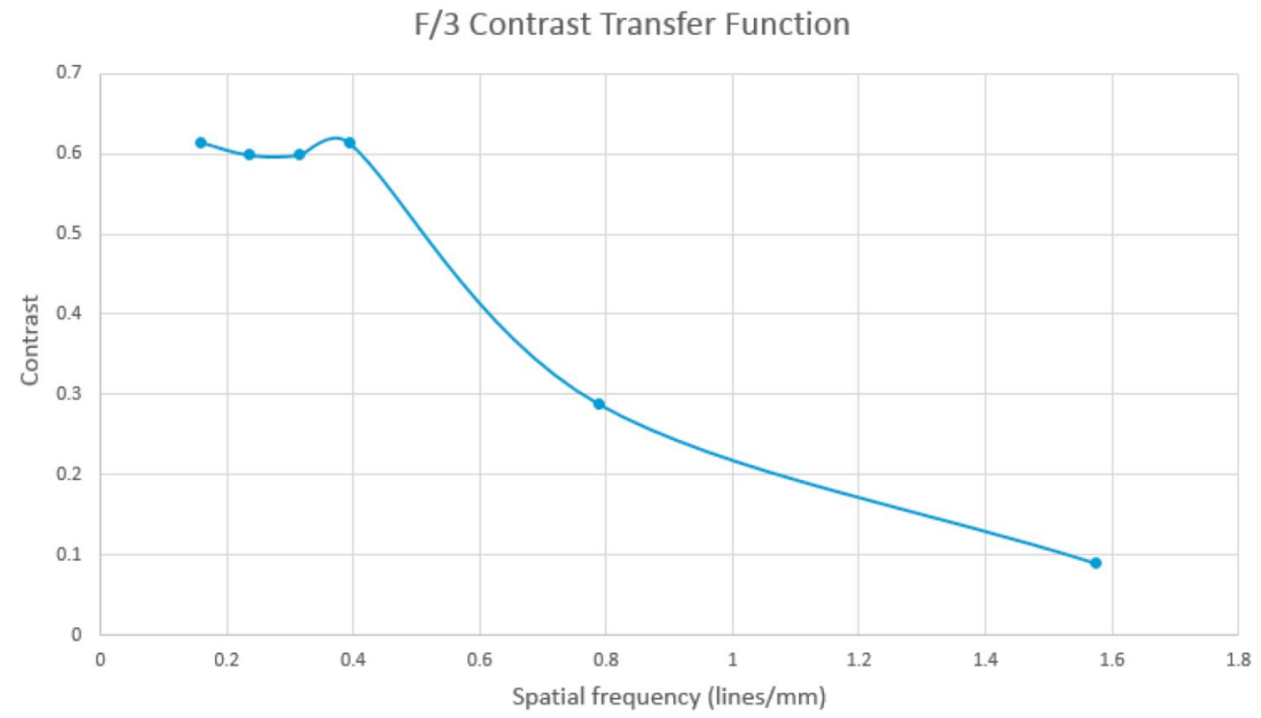

Figure 11 - (Project \#4 sample student work) Contrast transfer function for the compound camera lens generated using the data shown in Figure 9. Note horizontal axis scale incorrectly refers to the spatial frequency in the object plane (the contrast transfer function is typically specified as a function of spatial frequency in the image plane).

\section{CONCLUSION}

Evaluations of this course from this cohort of students were positive but also provided several constructive comments for improvement. This year we plan to better integrate the opto-mechanics topics from the lecture with the project schedule. For example, several students proposed using Foucault's method and we plan to try and incorporate this measurement as a second method for measuring the speed of light. This same student cohort just completed the first course of their capstone design experience during the spring quarter 2016 [2]. One observation noted by the course instructors was that every student team successfully demonstrated a working design for every project which had never been the case for past classes. Further assessment of student confidence in their ability and skills in the laboratory will be performed as this cohort of students completes their capstone design course sequence this academic year. 


\section{ACKNOWLEDGEMENTS}

The authors would like to thank Daniel K. Lauriola and Jacob D. Tyler for granting permission to use their coursework as examples of student submissions in this paper.

\section{REFERENCES}

[1] R. M. Bunch, C. H. Joenathan, and A. Z. Siahmakoun, "From optics to optical engineering: 20 years of optics education at Rose-Hulman Institute of Technology," presented at the Education and Training in Optics and Photonics, 2003.

[2] R. M. Bunch, P. O. Leisher, and S. C. Granieri, "Optical engineering capstone design projects with industry sponsors," Proc. of SPIE, vol. 9188, 2014.

[3] P. O. Leisher, S. C. Granieri, R. M. Bunch, A. Siahmakoun, and C. Joenathan, "Curriculum revisions for meeting the new ABET program-specific criteria in optical engineering," 2015, pp. 979321-979321-8.

[4] ABET Criteria for Accrediting Engineering Programs. Available: http://www.abet.org/wpcontent/uploads/2015/04/E001-14-15-EAC-Criteria.pdf

[5] www.thorlabs.com 